\title{
Prevalência de distúrbio ostemuscular e qualidade de vida em alunos do curso de fisioterapia
}

\author{
Prevalence of ostemuscular disorder and quality of life \\ of physiotherapy students
}

Ana Paula Nassif Tondato da Trindade, ${ }^{1}$ Givanir Renato de Almeida, ${ }^{1}$ Anderson Carvalho Santos, Fabrício Borges Oliveira'

'Centro Universitário do Planalto de Araxá (Uniaraxá), Araxá, MG, Brasil.

Recebido em: 26/07/2016 / Aceito em: 22/08/2016 / Publicado em: 18/10/2016

anapaulanassif@yahoo.com.br

\section{RESUMO}

Objetivo: avaliar a prevalência dos distúrbios osteomusculares e a qualidade de vida dos alunos do curso de fisioterapia do Uniaraxá. Método: trata-se de um estudo observacional, transversal, com 110 indivíduos, de ambos os gêneros na faixa etária de 18 a 45 anos, sendo todos alunos regulares do curso de fisioterapia de uma instituição de ensino superior. A coleta de dados foi iniciada com o preenchimento da ficha de avaliação, contendo informações sócio-demográficas, seguida pelo questionário SF-36, e finalmente pelo Questionário Nórdico de Sintomas Osteomusculares. Resultados: a idade média dos alunos foi de 20,9 anos. De acordo com o questionário Nórdico, a queixa mais frequente de dor nos últimos 12 meses foi na região da coluna lombar com 58,2\%. Já, nos últimos 7 dias a região álgica permanece a mesma porém com diminuição para $35,5 \%$ dos casos. De acordo com o questionário SF36 , pode-se perceber que a o menor valor encontrado foi no domínio vitalidade $(57,1 \%)$ e o maior valor para capacidade funcional (87,9\%). Considerações finais: observamos que os pacientes apresentaram uma boa qualidade de vida, sendo que o menor valor encontrado foi no domínio vitalidade. Há, porém, grande prevalência de distúrbios osteomusculares. Este fato indica a importância de se fazer avaliações periódicas e ver a necessidade de implantação de estratégias em promoção de saúde para melhorar a condição de hábitos de vida diária, ajudar no trabalho e nos estudos, minimizando a prevalência de distúrbios osteomusculares, como forma de prevenção, para que haja uma diminuição destes índices relacionados à dor.

Palavras-chave: Distúrbios Osteomuscular; Dor; Qualidade de vida.

\section{ABSTRACT}

Objective: to evaluate the prevalence of musculoskeletal disorders and quality of life of UNIARAXÁ physiotherapy course students. Method: this is an observational, cross-sectional study of 110 individuals of both genders aged 18-45 years, all regular physiotherapy students of a high school institution. Data collection was started with the completion of the evaluation form containing sociodemographic information, followed by the SF-36 questionnaire, and finally by the Nordic Musculoskeletal questionnaire. Results: mean age of students was 20.9 years. According to the Nordic questionnaire the most frequent complaint of pain in the last 12 months was in the lumbar spine region with 58.2\%. In the last seven days the painful area remains the same but with a reduction to $35.5 \%$ of cases. According to the SF-36 questionnaire, one can see that the lowest value was in the field vitality (57.1\%) and the highest value for functional capacity (87.9\%). Closing remarks: we observed that patients had a good quality of life and the smallest value was found in the field vitality. However, there is a high prevalence of musculoskeletal disorders. These indicates the importance of doing regular assessments and see the need to implement health promotion strategies; to improve the condition of daily life habits, help in work and studies, minimizing the prevalence of musculoskeletal disorders, in order to prevent so that there is a decrease of these indices related to pain.

Keywords: Musculoskeletal Disorders; Pain; Quality of life. 


\section{INTRODUÇÃO}

A dor osteomuscular é considerada multidimensional. Isso porque está relacionada a várias características como sensoriais, físicas, emocionais e biomecânicas. Tem uma relação universalmente ampla, podendo ser uma das causadoras de vários encaminhamentos de pacientes para os setores particulares ou públicos de saúde. ${ }^{1}$

A literatura comprova que doenças e limitações não são mais consequências inevitáveis e que o uso de serviços preventivos, eliminação de fatores de risco e adoção de hábitos de vida saudável são consideráveis determinantes para uma vida saudável. ${ }^{2}$

O aparecimento dos distúrbios osteomusculares tem crescido em graus mundiais e, quando envolvidos com serviço, podem ganhar diferentes terminologias, como distúrbio osteomuscular relacionado ao trabalho (DORT) ou lesão por esforço repetitivo (LER).

Deve-se ressaltar que as condições de prevenção referem-se à atenção aos riscos ambientais, ou seja, a todos os fatores de risco extrínsecos e a fatores pessoais que possam provocar doenças e que podem ou não serem alterados. Portanto, é possível que, desde a juventude, todos os indivíduos tenham se preocupado com a qualidade de vida, como a prática de atividade física, orientada uma alimentação adequada, não se tornar dependente químico nem possuir nenhum vicio nocivo ao corpo humano. ${ }^{3}$

A existência de um fator de risco no trabalho, em casa, na faculdade, entre outros, não é suficiente para o surgimento de algum distúrbio osteomuscular. Para que isso aconteça precisaria ser necessário um determinado grau de gravidade associado de outros fatores. Inúmeros fatores de risco envolvidos aos distúrbios osteomusculares podem não ocasionar diretamente alguma alteração, mas ajudam as reações fisiopatológicas que determinam seu surgimento. ${ }^{4}$

As alterações fisiológicas que ocorrem com o passar do tempo com as repetitividades do dia a dia não incluem alterações em decorrência de processos patológicos. As doenças de modo comum associada se devem a abusos de longo prazo, embora, não deve ser afirmado que todas as doenças podem ser evitadas por uma vida saudável. Uma compreensão das alterações fisiológicas e do seu impacto sobre a função é o primeiro passo para o desenvolvimento de medidas terapêuticas ou preventivas racionais para abordar esses problemas. ${ }^{5}$

$\mathrm{Na}$ literatura, é ressaltado que não existe um só fator biomecânico determinante. Os distúrbios osteomusculares podem ser também devido à repetitividade, ao excesso de cargas e posturas estáticas. ${ }^{6}$

Uma forma de se avaliar os distúrbios osteomusculares é através do questionário Nórdico. Esse questionário foi desenvolvido com a proposta de padronizar a mensuração de relato de sintomas osteomusculares e, assim, facilitar a comparação dos resultados entre os estudos. Os autores deste questionário não indicam como base para diagnóstico clínico, mas para identificação de distúrbios osteomusculares e, como tal, pode constituir importante instrumento de diagnóstico de ambiente ou do posto de trabalho. O questionário foi traduzido para diversos idiomas na última década, dando origem a muitos estudos empíricos. $\mathrm{O}$ instrumento consiste em escolhas múltiplas ou binárias quanto à ocorrência de sintomas nas diversas regiões anatômicas nas quais são mais comuns. ${ }^{7}$
É destacada a relevância cientifica e social de se investigar as condições que interferem no bem-estar e os fatores associados à qualidade de vida, a fim de criar alternativas de interação e propor ações e políticas na área da saúde, buscando atender as demandas da população. ${ }^{8}$ Hoje em dia se fala muito em qualidade de vida e essa caminha em lados opostos aos distúrbios osteomusculares, pois o individuo que tem um bom estilo de vida, dificilmente terá disfunções osteomusculares. ${ }^{9}$

Ao longo dos anos, juntamente com o avanço tecnológico e cientifico, há uma crescente busca da população mundial por uma melhor qualidade de vida. Esses fatores atrelados ao avanço da medicina tem aumentado a expectativa de vida da população. ${ }^{10} \mathrm{~A}$ saúde passa a ser vista como um recurso para a vida, e não como um objetivo de viver, portanto, a promoção da saúde não é responsabilidade exclusiva do setor de saúde, ela transcende este setor e vai além de um estilo de vida saudável; vai a direção de um bem-estar global. ${ }^{11}$

Para o ser humano ter uma boa qualidade de vida, podemos interpretar como um fato de que eles podem se sentir melhor, conseguindo cumprir com suas funções de vida diária básica adequadamente e conseguirem viver de forma independente. Além disso, o engajamento com a vida, a liberdade de doenças e a competência física e mental, são questões importantes na manutenção da qualidade de vida da população. ${ }^{12}$

A qualidade de vida da população está associada à manutenção da capacidade funcional. Deve-se considerar que a qualidade de vida também está relacionada à segurança socioeconômica, ao bem estar psicossocial e a saúde. Todos esses fatores são inter-relacionados e sua importância relativa difere de uma sociedade para outra e de pessoa pra pessoa. ${ }^{13}$

A qualidade de vida é entendida como a percepção que o individuo tem sobre sua posição na vida. Envolve aspectos culturais e valores nos quais ele está inserido. Contemplando a relação entre seus objetivos, esperanças, modelos e preocupações como forma de parâmetro. ${ }^{14}$ Torna-se, portanto, um grande desafio promover, avaliar e conhecer a real condição necessária para se ter uma boa qualidade de vida. ${ }^{15}$

Dentre os instrumentos utilizados para avaliação da qualidade de vida, podemos citar o SF-36. Este questionário foi traduzido e validado para a língua portuguesa, sendo constituído por 36 itens divididos em 8 domínios: capacidade funcional, aspectos físicos, dor, estado geral de saúde, vitalidade, aspectos sociais, aspectos emocionais e saúde mental. Este questionário apresenta um escore final de 0 a 100, no qual o 0 corresponde ao pior estado geral de saúde e 100 ao melhor estado de saúde. ${ }^{16}$

Esse questionário avalia, tanto aspectos negativos de saúde (doença ou enfermidade), como aspectos positivos (bem-estar). Os dados são avaliados a partir da transformação das respostas em escores escala de 0 a 100, de cada componente, não havendo um único valor que resuma toda a avaliação, resultando em um estado geral de saúde melhor ou pior. Cada escala (dimensão) recebe um escore que varia de 0 a 100, que corresponde do pior ao melhor estado de saúde. ${ }^{17}$ É um questionário genérico, com conceitos não específicos para uma determinada idade, doença ou grupo de tratamento e que permite comparações entre diferentes patologias e trata- 
mentos. O questionário é auto aplicável, de fácil execução e compreensão, considera a percepção dos indivíduos, quanto ao seu próprio estado de saúde e contempla os aspectos mais representativos da saúde funcional.

O resultado destes domínios é chamado de Raw Scale, tendo em vista que o valor final não apresenta nenhuma unidade em medida. ${ }^{16}$

Portanto, o objetivo desse estudo é avaliar a prevalência desses distúrbios e sua correlação com a qualidade de vida dos universitários do curso de fisioterapia a fim de demonstrar, se eles possuem ou não limitações funcionais e se esses distúrbios podem ou não estarem relacionados à qualidade de vida.

\section{MÉTODO}

O estudo foi previamente aprovado pelo CEP Uniaraxá sob o protocolo $N^{\circ} 43994-38$ e os pacientes foram informados sobre os procedimentos e assinaram um termo de consentimento livre e esclarecido, conforme apêndice II, de acordo com a resolução 466/2012 do Conselho Nacional de Saúde, resolução que normatiza a pesquisa com seres humanos.

Para realização do estudo, os processos realizados obedeceram os principios norteadores da bioética, resguardando o anonimato, a autonomia, não maleficência e assegurando os direitos e deveres do pesquisador e dos sujeitos pesquisados.

Os participantes do projeto foram previamente orientados sobre os procedimentos do estudo e, após o esclarecimento das informações sobre a pesquisa, as sinaram um termo de consentimento livre e esclarecido confirmando sua participação. A coleta de dados foi realizada entre os períodos de maio a setembro de 2014 pelo questionário nórdico e SF-36, nas dependências do campus do Uniaraxá, Araxá - MG.

O tamanho da amostra foi calculado considerando a um intervalo de confiança de $95 \%$ com alfa de $5 \%$. De acordo com o levantamento realizado junto à secretaria acadêmica, haviam 130 alunos matriculados. Portanto, de acordo com os cálculos amostrais deveriam ser avaliados no mínimo 109 alunos que se encaixassem nos critérios de inclusão.

Foram inclusos a este trabalho, universitários do Uniaraxá, de ambos os gêneros, na faixa etária entre 18 e 45 anos, que estavam cursando, regularmente, o curso de fisioterapia e que concordaram em participar da pesquisa, assinando o termo de consentimento livre e esclarecido. Foram excluídos deste trabalho acadêmicos do Uniaraxá dos demais cursos, ou que apresentassem alguma alteração osteomuscular prévia.

A pesquisa teve início com o levantamento sócio-demográfico dos estudantes do curso de fisioterapia do Uniaraxá, com questões pessoais como idade, sexo, estado civil, número de filhos, etilismo, se trabalha e a carga horária (em caso afirmativo).

Em seguida, como forma de averiguar de forma positiva ou negativa a qualidade de vida funcional dos participantes, evidenciando as suas reais necessidades, quanto à melhoria da sua qualidade de vida na realização de atividades funcionais da vida diária e profissional, foi aplicado o Questionário de Qualidade de Vida SF -36.

A coleta de dados terminou com a avaliação do grau de dor utilizando o Questionário Nórdico de Sintomas Osteomusculares (QNSO). O questionário nórdico (QNSO) foi desenvolvido com a proposta de padronizar a mensuração de relato de sintomas osteomusculares e, assim, facilitar a comparação dos resultados entre os estudos.

As pontuações correspondentes a cada item, descrito pelas avaliações, foram assinaladas e, posteriormente, somadas para a verificação da pontuação total de cada escala.

A análise dos dados foi realizada através de estatística descritiva, em que se calculou a média, o desvio padrão, porcentagem, maior valor e menor valor para as variáveis obtidas no questionário sócio-demográfico, nórdico e no SF-36. Os resultados foram apresentados em forma de gráficos e tabelas.

\section{RESULTADOS}

A amostra foi composta de 110 voluntários que responderam aos questionários, após assinarem o termo de consentimento livre e esclarecido, ficando ca-

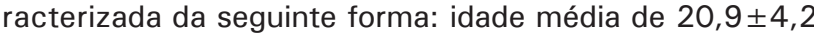
anos, com idade mínima de 18 anos e máxima de 45 anos.

$\mathrm{Na}$ Tabela 1, estão representadas as características da amostra, considerando o perfil sociodemografico evidenciando os seguintes aspectos: idade (em anos), sexo, se possui filhos, o estado civil, se pratica ou não atividade física e se tem ocupação laboral.

Tabela 1 - Resultados do Perfil Sociodemografico dos Universitários do Curso de Fisioterapia, contendo as seguintes características: idade (em anos), sexo, filhos, estado civil, praticante de atividade física, ocupação laboral (Araxá, 2014)

\begin{tabular}{llcc}
\hline Característica & Categoria & $\mathbf{N}^{\mathbf{0}}$ & $\%$ \\
\hline Idade (em anos) & Até 19 & 25 & 22,7 \\
& De $20-25$ & 71 & 64,5 \\
& De 26 - 30 & 9 & 8,2 \\
& Acima de 30 & 5 & 4,5 \\
\hline Sexo & Masculino & 21 & 19,1 \\
& Feminino & 89 & 80,9 \\
\hline Filhos & Sim & 13 & 11,8 \\
& Não & 97 & 88,2 \\
\hline Estado civil & Solteiro & 101 & 91,9 \\
& Casado & 9 & 8,1 \\
\hline Praticante de Atividade física & Sim & 51 & 46,3 \\
& Não & 59 & 53,7 \\
\hline Ocupação laboral & Sim & 45 & 40,9 \\
& Não & 65 & 59,1 \\
\hline Total & & 110 & 100 \\
\hline
\end{tabular}

Fonte: Dados da pesquisa (2014).

Os resultados obtidos pelo questionário Nórdico apontam que as queixas mais frequentes de dor, nos últimos 12 meses, foram na região da coluna lombar com 58,2\% dos casos. Já, nos últimos 7 dias a região álgica permanece a mesma, porém com diminuição para $35,5 \%$ dos casos, como mostra a tabela 2 .

De acordo com o Questionário SF-36, a maioria dos entrevistados possui uma boa qualidade de vida, pois em todos os quesitos avaliados o menor valor encontrado foi vitalidade com $57,1 \%$ dos casos, como mostra a tabela 3 . 
Tabela 2 - Resultados da distribuição dos distúrbios osteomusculares relacionados às região do corpo, nos últimos 7 dias e nos últimos 12 meses (Araxá, 2014).

\begin{tabular}{lcccc}
\hline Região & \multicolumn{2}{c}{ Dor nos últimos } & \multicolumn{2}{c}{ Dor nos últimos } \\
& \multicolumn{2}{c}{ 12 } & meses & \multicolumn{2}{c}{$\mathbf{7}$ dias } \\
& N $^{\circ}$ & \% & No $^{\circ}$ & $\%$ \\
\hline Pescoço & 56 & 50,9 & 56 & 50,9 \\
Ombro & 37 & 33,6 & 37 & 33,6 \\
Dorsal & 41 & 37,3 & 41 & 37,3 \\
Cotovelo & 6 & 5,5 & 6 & 5,5 \\
Antebraço & 12 & 10,9 & 12 & 10,9 \\
Lombar & 64 & 58,2 & 64 & 58,2 \\
Punhos/mãos/dedos & 30 & 27,3 & 30 & 27,3 \\
Quadris e coxas & 26 & 23,6 & 26 & 23,6 \\
Joelho & 30 & 27,3 & 30 & 27,3 \\
Tornozelo/pés & 33 & 30 & 33 & 30 \\
\hline
\end{tabular}

Fonte: Dados da pesquisa (2014).

Tabela 3 - Resultados dos aspectos avaliados para se determinar qualidade de vida dos universitários do curso de Fisioterapia (Araxá, 2014).

\begin{tabular}{lcccc}
\hline Perfil Sociodemografico & Media & $\begin{array}{c}\text { Dor } \\
\text { Padrão }\end{array}$ & $\begin{array}{c}\text { Maior } \\
\text { Valor }\end{array}$ & $\begin{array}{c}\text { Menor } \\
\text { Valor }\end{array}$ \\
\hline Capacidade funcional & 87,9 & 11,7 & 100 & 55 \\
Limitação por aspectos físicos & 75,4 & 31,2 & 100 & 0 \\
Dor & 62,3 & 19,5 & 100 & 10 \\
Estado geral de saúde & 62,3 & 15,1 & 100 & 20 \\
Vitalidade & 57,1 & 19,4 & 100 & 5 \\
Aspectos sociais & 72,1 & 22,5 & 100 & 25 \\
Aspectos emocionais & 73,6 & 35,6 & 100 & 0 \\
Saúde mental & 66,4 & 18 & 100 & 12 \\
\hline Fonte:
\end{tabular}

Fonte: Dados da pesquisa (2014).

\section{DISCUSSÃO}

Vários estudos caracterizam a população universitária brasileira do gênero feminino, solteira, sem filhos, com idade média em torno de 21 anos, morando com os pais e sem um trabalho remunerado. Podemos observar esses dados em um estudo, ${ }^{18}$ na universidade pública do Nordeste; em que obtiveram a idade média 20,6 anos; assim como em outro estudo, ${ }^{19}$ a maioria dos universitários são do sexo feminino $(65,9 \%)$, e com mesma faixa etária. Outro estudo ${ }^{20}$ mostra que a média de idade observada foi de 22,3 anos, sendo uma pequena maioria do gênero feminino (55,9\%). Esse mesmo estudo aponta que a maioria dos alunos morava com a família $(75,1 \%)$, eram solteiros $(90,6 \%)$, não tinham filhos $(92,6 \%)$, não realizavam trabalho remunerado além da universidade $(60,5 \%)$ e tinham suas despesas custeadas pelos pais $(57,7 \%)$. Em todos esses estudos os resultados se assemelham ao do nosso estudo, mostrando que a população acadêmica tem caraterísticas sócio demográficas semelhantes independente da região estudada. Esse fato pode ser justificado pelo fato dos alunos encerrarem o ensino médio e já ingressarem em algum curso de graduação. ${ }^{21}$

Por esses dados podemos presumir que a população feminina busca se capacitar mais para o mercado de trabalho e adia a formação da família e a maternidade. Esses dados evidenciam também a dependência financeira dos filhos, que tem suas despesas custeadas pelos pais.

As mulheres na área da saúde, tem um predomínio quase total, assim como no presente estudo, este predomínio pode ser justificado pelo fato de que as mulheres possuem maior cuidado e atenção com a saúde, quando comparadas aos homens, que por sua vez prevalecem nas áreas de engenharias e ciências exatas. ${ }^{22}$

A prática da atividade física é benéfica à saúde e por isso deve ser incentivada no ambiente universitário. Sabe-se que os estudantes estão continuamente sujeitos a situações de estresse como elaboração de trabalhos, prazos de entrega, longas horas de estudo e situações de exaustão física e mental. Pesquisa com universitários croatas identificou que a realização de programas de esporte nos tempos livres diminui as referências a desconfortos, em relação à sua saúde. ${ }^{18}$

Os resultados do presente estudo demonstraram que as queixas de distúrbios osteomusculares estavam menores dentre os universitários de fisioterapia nos últimos sete dias comparado aos resultados encontrados nos últimos 12 meses. Esses achados indicam principalmente que as alterações crônicas estão mais presentes nos alunos do curso de fisioterapia. A região lombar foi à região anatômica mais acometida, nos dois períodos (últimos 7 dias e últimos 12 meses). Esse resultado aponta a lombalgia como o problema mais comum entre os alunos, apresentado, tanto um aspecto agudo, como crônico. As outras regiões mais afetadas nos últimos 12 meses como nos últimos 7 dias foram a região de pescoço e a região dorsal.

Num estudo realizado em Belo Horizonte em estudantes de fisioterapia, os autores que observaram a queixa de dor lombar maior responsável por $63,1 \%$ do total de queixas, seguido da cervical $(47,3 \%) .{ }^{23}$

Embora a lombalgia apresente relação com as condições de trabalho, a alta prevalência de dor na coluna, não é uma característica única dos universitários de fisioterapia, mas é um fato que afeta diversas profissões e a população em geral. ${ }^{24}$ Os distúrbios da coluna vertebral são de origem multifatorial e podem envolver fatores psicossociais, inatividade física e obesidade. Também pode se entender que a elevada prevalência de desconforto na região lombar justifica-se pela postura sentada, que a maioria dos alunos utilizam em sala de aula, tanto quanto em casa, na frente do computador e ainda em alguns casos por se deslocarem de outras cidades para esudar. ${ }^{25}$ A posição sentada aumenta a compressão intra-discal e faz com que a coluna receba uma carga mecânica maior do que na posição de pé, apresentando uma maior probabilidade de desconforto ou dor na região lombar, tal como evidencia este estudo. ${ }^{26}$

Essas alterações podem estar associadas a vários fatores são predisponentes para a lombalgia; alguns intrínsecos como a idade, obesidade, gênero e raça, outros extrínsecos como fatores ergonômicos, sedentarismo, sobrecarga muscular. ${ }^{27}$ Além desses fatores, observa-se que a escolaridade e a atividade laboral também são fatores de risco para o agravo.

Na perspectiva de qualidade de vida, como o completo bem estar físico, psíquico e social e como a manutenção do equilíbrio entre a vida social e as atividades profissionais, pode-se considerar que os desequilíbrios gerados no ambiente de trabalho no dia a dia podem afetar a qualidade de vida de um indivíduo. ${ }^{28}$ 
No presente estudo, pode-se perceber que a maioria dos entrevistados possui uma boa qualidade de vida, pois em todos os quesitos avaliados o menor valor encontrado foi vitalidade com $57,1 \%$ e a maior capacidade funcional com $87.9 \%$ dos casos. Com isso, podemos considerar assim que dentre os alunos de fisioterapia do Uniaraxá, seu estilo de vida e sua prática de exercícios é bem regular.

Estudos nacionais com estudantes de medicina com objetivo de avaliar a qualidade de vida desses alunos também registram altos níveis de percepção do nível de qualidade de vida. ${ }^{29,30}$ Outro estudo realizado com alunos de fisioterapia não encontrou esse resultado mostrando que a dor influencia na qualidade de vida do individuo. ${ }^{31}$

Um estudo analisando a qualidade de vida de estudantes de enfermagem, os autores verificaram que $72 \%$ dos participantes da sua pesquisa, classificaram sua qualidade de vida como "boa" ou "muito boa" e $67,7 \%$ estavam satisfeitos com sua saúde, assim como no presente estudo ${ }^{32}$. Esses estudos permitem considerar que grande parte dos estudantes de curso superior caracterizam sua saúde como boa ou muito boa.

\section{CONSIDERAC̣ÕES FINAIS}

Perante os achados do presente estudo foi possível observar uma alta prevalência de sintomas osteomusculares nos últimos doze meses e nos últimos sete dias, com principal envolvimento da região lombar, cervical e região dorsal, respectivamente, mostrando ser em sua maioria alteração crônica. Pode-se perceber também que a maioria dos entrevistados possui uma boa qualidade de vida, pois em todos os quesitos avaliados o menor valor encontrado foi vitalidade com $57,1 \%$ dos casos.

De acordo com esses dados sugere-se que os resultados desta pesquisa demonstraram que a maioria dos estudantes possui altos índices na percepção de qualidade de vida, porém há grande prevalência de distúrbios osteomusculares. Com isso é de suma importância se fazer avaliações periódicas e ver a necessidade de implantação de estratégias em promoção de saúde para melhorar a condição de hábitos de vida diária, ajudar no trabalho e nos estudos, minimizando a prevalência de distúrbios osteomusculares, como forma de prevenção, para que haja uma diminuição destes índices relacionados à dor.

\section{AGRADECIMENTOS}

Agradecemos ao Centro Universitário do Planalto de Araxá pelo apoio recebido durante a realização dessa pesquisa.

\section{REFERÊNCIAS}

1. Paixão MS, Tassitano RM, Siqueira GR. Prevalência de desconforto osteomuscular e fatores associados em estudantes universitários. Rev Bras Promoç Saúde, Fortaleza 2013;26(2):242-250.

2. Jannini SN, Filho UD, Damiani D, Silva CAA. Dor músculo-esquelética em adolescentes obesos. J Pediatr.
2011;87(4):329-335. doi: 10.2223/JPED.2111.

3. Santos FH, Andrade VM, Bueno OFA. Envelhecimento: Um Processo Multifatorial. Psicol Est, Maringá 2009;14(1):3-10. doi: 10.1590/S1413-73722009000100002

4. Maeno M, Almeida IM, Martins MC, Toledo LF, Paparelli R, Silva JAP. Lesões por esforços repetitivos (LER) distúrbios osteomusculares relacionados ao trabalho (DORT). Ministério Da Saúde, Brasília, Serie A. Normas e Manuais Técnicos 2001;103:0-36

5. Pedott AH, Fogliatto FS. Estudos de repetitividade e reprodutividade para dados funcionais. Produção 2013;23(3):548-60.

6. Caetano VC, Cruz DT, Leite ICG. Perfil dos pacientes e características do tratamento fisioterapêutico aplicado aos trabalhadores com LER/DORT em Juiz de Fora, MG. Fisioterapia do Movimento, Curitiba 2010;23(3):451-460.

7. Pinheiro FA, Tróccoli BT, Carvalho CV. Validação do questionário nórdico de sintomas osteomusculares como medida de morbidade. Rev Saúde Públ, Brasília 2002;36(2):307-312.

8. Fleck MPA, Lima AFBS, Louzada S, Schestasky G, Henriques A, Borges VR, Camey Suzi, Lido G. Associação entre sintomas depressivos e funcionamento social em cuidados primários à saúde. Rev Saúde Públ 2002;36(4):431-438. doi: 10.1590/S0034-89102002000400008.

9. Gimenes GF. Uso e significados da qualidade de vida nos discursos contemporâneos de saúde. Trab Educ Saúde, Rio De Janeiro 2013;11(2):291-318. doi: 10.1590/S198177462013000200003.

10. Araujo DSMS, Araujo CGS. Aptidão física, saúde e qualidade de vida relacionada à saúde em adultos. Rev Bras Med Esporte. 2000;6(5):194-203. doi: 10.1590/S151786922000000500005

11. 11. Buss PM. Promoção da saúde e qualidade de vida. Ciênc Saúde Coletiva 2000;5(1):163-177. doi: 10.1590/S141381232000000100014

12. Seidl EMF, Zannon CMLC. Qualidade de vida e saúde: aspectos conceituais e metodológicos. Cad Saúde Públ, Rio de Janeiro 2005;2(20):580-588.

13. Gaspar T, Matos MG, Ribeiro JLP, Leal I. Qualidade de vida e bem-estar em crianças e adolescentes. Rev Bras Ter Cogn Rio De Janeiro 2006;2(2)491-500.

14. Schlosser TCM, Ceolim MF. Qualidade de vida de pacientes com câncer no período de quimioterapia. Texto contexto enferm, Florianópolis 2012;21(3):600-607.

15. Teixeira CF, Paim JS. Planejamento e programação de ações intersetoriais para a promoção da saúde e da qualidade de vida. RAP, Rio De Janeiro 2000;34(6):63-80.

16. Ciconelli RM, Ferraz MB, Santos W, Meinão I, Quaresma MR. Tradução para a língua portuguesa e validação do questionário genérico de avaliação de qualidade de vida SF36. Rev Bras Reumatol, São Paulo 1999;39(3):143-150.

17. Martinez MC, Paraguay AIBB, Latorre MRDO. Relação entre satisfação com aspectos psicossociais e saúde dos trabalhadores. Rev Saúde Pública 2004;38(1):55-61. doi: 10.1590/S0034-89102004000100008.

18. Silva DAS, Pereira IMM, Almeida MB, Silva RJS, Oliveira ACC. Estilo de vida de acadêmicos de educação física de uma universidade publica do Estado de Sergipe. Rev Bras Ciênc Esporte, Florianópolis 2012;34(1):53-67. doi: 10.1590/ S0101-32892012000100005.

19. Silva AO, Neto JLC. Associação entre níveis de atividade física e transtorno mental comum em estudantes universitários. Motri 2014;10(1):49-59. doi: 10.6063/motricidade.10(1).2125. 
20. Fontes ACD, Vianna RPT. Prevalência e fatores associados ao baixo nível de atividade física entre estudantes universitários de uma universidade pública da região Nordeste - Brasil. Rev Bras Epidemiol 2009;12(1):20-29. doi: 10.1590/S1415790X2009000100003.

21. Souza LAF, Silva CD, Ferraz GC, Sousa FAF, Pereira LV. Prevalência e caracterização da prática de automedicação para alívio da dor entre estudantes universitários de enfermagem. Rev Latino-Am Enfermagem 2011;19(2):02-07. doi: 10.1590/S0104-11692011000200004.

22. Leite ACB, Grillo LP, Caleffi F, Mariath AB, Stuker H. Qualidade de vida e condições de saúde de acadêmicos de nutrição. Revista Espaço para a Saúde, Londrina 2011;13(1):82-90.

23. Silva FFD, Marçal MA, Nicácio AS. Avaliação da prevalência de lombalgia em estudantes de fisiocterapia durante estagio ambulatorial. BJPT 2007;11(Suppl):203-203.

24. Serranheira F, Cotrim T, Rodrigues V, Nunes C, Uva AS. Lesões musculoesqueléticas ligadas ao trabalho em enfermeiros portugueses: ou doenças relacionadas com o trabalho? Rev Port Sau Pub 2012;30(2):193-203. doi: 10.1016/j.rpsp.2012.10.001.

25. Helfenstein Junior M, Goldenfum M, Siena C. Lombalgia Ocupacional. Rev Assoc Med Bras 2010;56(5):583-589.

26. Hobner AR, Mendes MR, Queruz JCF, Dambrós JM, Suaréz ADH, Spinelli LF. Avaliação do tratamento da discopatia degenerativa cervical pela artrodese via anterior utilizando placas associadas A Cages ou Cages em Peek Isoladamente. Coluna/Columna 2011;10(4):300-304. doi: 10.1590/S1808-

\section{0}

27. Duarte AF, Souza APC, Macedo AF, Araujo FF, Passos JP, Pereira CA. Fatores de riscos para distúrbios osteomusculares relacionados ao trabalho-DORT em profissionais de enfermagem. R pesq cuid Fundam Online 2012;(Ed. Supl.):53-56.

28. Rocha ALS. Analise ergonômica do colete de proteção balística utilizado pela Policia Militar do Estado de São Paulo. 2009. Disponível em: <Http://Www.Policiamilitar.Sp.Gov. $\mathrm{Br} /$ Caes/Artigos/Artigos\%20pdf/Afonso\%20Luiz\%20 Sanches\%20Rocha.Pdf.>

29. Dias JCR, Libardi MC, Zillo CM, Igarashi MH, Senger $\mathrm{MH}$. Qualidade de vida em cem alunos do curso de medicina de Sorocaba-PUC/SP. Rev Bras Educ Med. Rio De Janeiro 2010;34(1):116-123. doi: 10.1590/S010055022010000100014.

30. Meyer C, Guimarães ACA, Machado Z, Parcias SR. Qualidade de vida e estresse ocupacional em estudantes de medicina. Rev Bras Educ Med. Rio De Janeiro 2012;36(4):489-498. doi: 10.1590/S0100-55022012000600007.

31. Bacchi CA, Candotti CT, Noll M, Minossi CES. Avaliação de qualidade de vida da dor nas costas da funcionalidade e alterações da coluna vertebral de estudantes de fisioterapia. Motriz Rev Educ Fís. Rio Claro 2013;19(2):243-251. doi: 10.1590/S1980-65742013000200001.

32. Saupe R, Neitche EA, ME Cestari, Giorgi MDM, Krahl M. Qualidade de vida dos acadêmicos de enfermagem. Rev Latino-Am Enferm 2004;12(4):636-642. doi: 10.1590/ S0104-11692004000400009.

Como citar: TRINDADE, Ana Paula Nassif Tondato da et al. Prevalência de distúrbio ostemuscular e qualidade de vida em alunos do curso de Fisioterapia. Cinergis, Santa Cruz do Sul, v. 17, n. 4, out. 2016. ISSN 2177-4005. Disponível em: <https:// online.unisc.br/seer/index.php/cinergis/article/view/8054>. Acesso em: 11 out. 2016. doi:http://dx.doi.org/10.17058/cinergis. 17 i3. 8054 